Original Article

\title{
SODIUM ALGINATE-LOCUST BEAN GUM IPN HYDROGEL BEADS FOR THE CONTROLLED DELIVERY OF NIMESULIDE-ANTI-INFLAMMATORY DRUG
}

\author{
C. MADHAVI ${ }^{1}$, P. KUMARA BABU ${ }^{1}$, Y. MARUTHI ${ }^{1}$, A. PARANDHAMA ${ }^{1}$, O. SREEKANTH REDDY ${ }^{2}$, \\ K. CHOWDOJI RAO ${ }^{*}$, M. C. S. SUBHA ${ }^{2}$ and R. JEEVAN KUMAR ${ }^{3}$
}

\author{
${ }^{1}$ Deparment of Polymer Science and Tech, ${ }^{2}$ Department of Chemistry, ${ }^{3}$ Department of Physics, Sri Krishnadevaraya University, \\ Anantapuramu, Andhra Pradesh, India \\ Email: chowdojirao@gmail.com
}

Received: 25 May 2017 Revised and Accepted: 31 Aug 2017

\begin{abstract}
Objective: The objective of this study was to formulate and evaluate the drug release studies using locust bean gum (LBG) and sodium alginate ( $\mathrm{NaAlg}$ ) and cross-linked with glutaraldehyde for the controlled release (CR) of Nimesulide, an anti-inflammatory drug.

Methods: Locust bean gum (LBG) and sodium alginate (NaAlg) blend hydrogel beads were prepared by an extrusion method using glutaraldehyde as a crosslinker. Nimesulide an anti-inflammatory drug was encapsulation within LBG/NaAlg blend hydrogel beads. Morphology, size, encapsulation efficiency and drug release from these hydrogel beads were evaluated by different characterization techniques such as fourier transform infrared spectroscopy (FTIR), differential scanning calorimetry (DSC), scanning electron microscopy (SEM), x-ray diffraction (X-RD) studies.

Results: Drug-loaded hydrogel beads were analyzed by FTIR, which indicates the interaction between drug and polymers. DSC thermograms on drug-loaded microbeads confirmed the polymorphism of Nimesulide and indicated a molecular level dispersion of the drug in the hydrogel beads. SEM confirmed the spherical nature and rough surface of the hydrogel beads produced. X-RD study was performed to understand the crystalline nature of drug after encapsulated into the hydrogel beads and confirmed the complete dispersion of the drug in the polymer matrix. In vitro release studies conducted in pH-7.4 which indicated a dependence of release rate on the amount of drug loading and the amount of LBG/NaAlg, but slow release rates were extended up to $48 \mathrm{~h}$. The cumulative release data were fitted to an empirical equation to compute diffusion exponent (n) which indicated the non-fickian trend for drug release.
\end{abstract}

Conclusion: These results clearly demonstrated that the ability of these newly developed hydrogel beads containing Nimesulide for its sustained release could possibly be advantageous to patient compliance with reduced dosing interval.

Keywords: Sodium alginate (NaAlg), Locust bean gum (LBG), Glutaraldehyde (GA) and Nimesulide

(c) 2017 The Authors. Published by Innovare Academic Sciences Pvt Ltd. This is an open-access article under the CC BY license (http://creativecommons.org/licenses/by/4.0/) DOI: http://dx.doi.org/10.22159/ijpps.2017v9i10.20231

\section{INTRODUCTION}

Carbohydrate polymers are extensively used in recent years in biomedical and pharmaceutical applications due to their biocompatibility and biodegradability [1]. Biopolymeric hydrogels can be prepared as three-dimensional hydrophilic networks that are capable of imbibing large quantities of water or biological fluids and release drugs at the controlled rates. Such networks can be composed of homopolymers or copolymers and are insoluble in water because of the presence of chemical or physical crosslinks such as entanglements or crystallites [2, 3]. Polymeric hydrogels have been studied in a variety of areas such as in chemical engineering, medicine, pharmaceuticals, food and agriculture [4-7]. However, the use of natural carbohydrate polymers like polysaccharides and proteins for biomedical applications has attracted the attention of many investigators [8-10]. Such naturally abundant carbohydrate polymers even though exhibit some limitations in their reactivity and processability have been still used after being modified by crosslinking, blending, etc. Many studies have been made in the literature to overcome this shortcoming by chemical and physical alterations of such natural carbohydrate polymer. Among these, development of hydrogels and interpenetrating polymer network (IPN) structures has received greater attention $[11,12]$. The successful formulation of a stable and effective dosage form, therefore, depends on the careful selection of natural polymers such as gums and other polymeric materials.

Alginates have been used as matrix forming material in the design of various drug delivery systems to achieve sustained drug release over a prolonged period due to their hydrogelforming properties [13]. On the other hand, locust bean gum is also derived from the endosperm of the seed of ceretonia siliqua linn belonging to the family fabaceae [14]. Locust bean gum has a wide potential in drug formulation due to its extensive application as a food additive and its recognized lack of toxicity. It can be tailored made to suit the demands of applicants in both the pharmaceutical and biomedical areas. This group of polymers possesses several characteristics that make it useful as a formulation aid, both as a conventional excipient and more specifically as a tool in polymeric controlled drug delivery. It consists mainly of a neutral galactomanan polymer made up of 1,4-linked D-mannopyronosyl units and in every fourth or fifth chain unit is substituted on C6 with a D-galactopyranosyl unit [15].

Nimesulide is a potential nonsteroidal anti-inflammatory drug known to provide better activity profile, greater safety and higher therapeutic index [16]. Nimesulide belongs to the class-II biopharmaceutical classification drug with a low solubility and high permeability $[17,18]$. Nimesulide is a weak inhibitor of prostaglandin synthesis in vitro and it appears to show its effects by a variety of mechanisms like free-radical scavenging, involving in neutrophil myeloperoxidase pathway, phosphodiesterase type IV inhibition, histamine release, tumor necrosis factor-alpha release, cartilage degradation, bradykinin activity, metaloprotease synthesis, platelet aggregation, and synthesis of platelet activity factor $[19,20]$ used the Nimesulide in control drug delivery $[21,22]$. Nimesulide produces gastric irritation in some cases and shows loss of its inhibitory cyclooxygenase-2 (COX-2) selectivity $[23,24]$. Nimesulide is sparingly soluble in water $(0.01 \mathrm{mg} / \mathrm{ml})$. This poor aqueous solubility would create difficulties in pharmaceutical formulations for oral and parenteral delivery that in turn may lead to variable bioavailability [25]. To overcome some of these drawbacks, we have planned the blend hydrogels of NaAlg and LBG by encapsulating with Nimesulide for drug release in a controlled manner as there 
were no reports on the use of these blends for drug delivery application.

In continuation of our ongoing research work on drug delivery studies [25-29], these hydrogel beads were characterized by FTIR SEM, X-RD, and DSC. The dissolution experiments were performed to study the drug release characteristics of the hydrogels and the results are presented here.

\section{MATERIALS AND METHODS}

\section{Materials}

Locust bean gum and Nimesulide were purchased from sigma aldrich chemical company, Milwankee, WI, USA. Sodium alginate (viscosity: $395 \mathrm{cps}$ for a $5 \%$ solution) was purchased from M/S moly chemicals, Mumbai (India). Analytical reagent grade glutaraldehyde (GA) [aqueous solution $25 \%(\mathrm{v} / \mathrm{v})$ ] and calcium chloride $\left(\mathrm{CaCl}_{2}\right)$ were purchased from Sd. Fine chemicals, Mumbai, India. Water used was of high purity grade after double distillation.
Preparation of sodiumalginate-locust bean gum hydrogel beads and drug loading

The hydrogel beads used for the study were prepared by extrusion of the mixture through a syringe having a diameter of $0.1 \mathrm{~mm}$ with different alginate to locust bean gum percentage weight ratios (3.75:0.25, 3.5:0.5, 3.25:0.75, 3:1, 2.75:1.25). The locust bean gum solution of the required concentration is prepared first and then the required amount of alginate is added and stirred well to form a uniform mixture. To this mixture, glutaraldehyde was added to a final concentration followed by Nimesulide with different variations and blended well. The final solution is kept undisturbed for some time to remove the trapped air bubbles. Beads are made in $0.5 \mathrm{M} \mathrm{CaCl} 2$ solution, cured for $1 \mathrm{~h}$ in the same solution and removed by filtration and washed with deionized water to remove excess glutaraldehyde and $\mathrm{CaCl}_{2}$. The beads were then either lyophilized or air-dried at room temperature and then stored in the refrigerator. The different formulation of hydrogel beads is given in table 1.

Table 1: The percentage of polymers, drug (Nimesulide) and cross-linked for the preparation of different Nimesulide-loaded hydrogel beads

\begin{tabular}{|c|c|c|c|c|c|}
\hline $\begin{array}{l}\text { Formulation } \\
\text { code }\end{array}$ & $\begin{array}{l}\text { Sodium alginate } \\
(\% \mathrm{w} / \mathrm{v})\end{array}$ & $\begin{array}{l}\text { LBG } \\
(\% \mathrm{w} / \mathrm{v})\end{array}$ & $\begin{array}{l}\text { Glutaraldehyde } \\
(\mathrm{ml})\end{array}$ & (\%) of drug loaded & $\begin{array}{l}\text { Encapsulation } \\
\text { efficiency }(\%) \pm S D\end{array}$ \\
\hline A1 & 3.75 & 0.25 & 1.5 & 5 & $42.58 \pm 0.6$ \\
\hline $\mathrm{A} 2$ & 3.5 & 0.5 & 1.5 & 5 & $53.25 \pm 1.2$ \\
\hline A3 & 3.25 & 0.75 & 1.5 & 5 & $49.87 \pm 0.5$ \\
\hline A4 & 3.0 & 1.0 & 1.5 & 5 & $71.63 \pm 0.7$ \\
\hline A5 & 3.0 & 1.0 & 1.5 & 10 & $74.23 \pm 0.5$ \\
\hline A6 & 3.0 & 1.0 & 1.5 & 15 & $63.98 \pm 0.8$ \\
\hline A7 & 3.0 & 1.0 & 1 & 5 & $61.03 \pm 1.1$ \\
\hline A8 & 3.0 & 1.0 & 2 & 5 & $68.59 \pm 0.8$ \\
\hline
\end{tabular}

\section{Fourier transform infrared (FTIR) spectral measurements}

FTIR spectral data were obtained using a Nicolet (Model Impact 410 Milwaukee, WI) instrument to confirm the formation of IPN structure and to find out any possible chemical interactions present among the polymers, drug and crosslinker.
For FTIR measurements, samples were crushed with $\mathrm{KBr}$ to make pellets using $600 \mathrm{~kg} / \mathrm{cm}^{2}$ pressure. FTIR spectra of placebo IPN hydrogel beads, drug-loaded blend beads, and plain Nimesulide were all scanned between 4000 and $500 \mathrm{~cm}^{-1}$. The probable schematic representation of IPN hydrogel beads is shown in fig. 1.

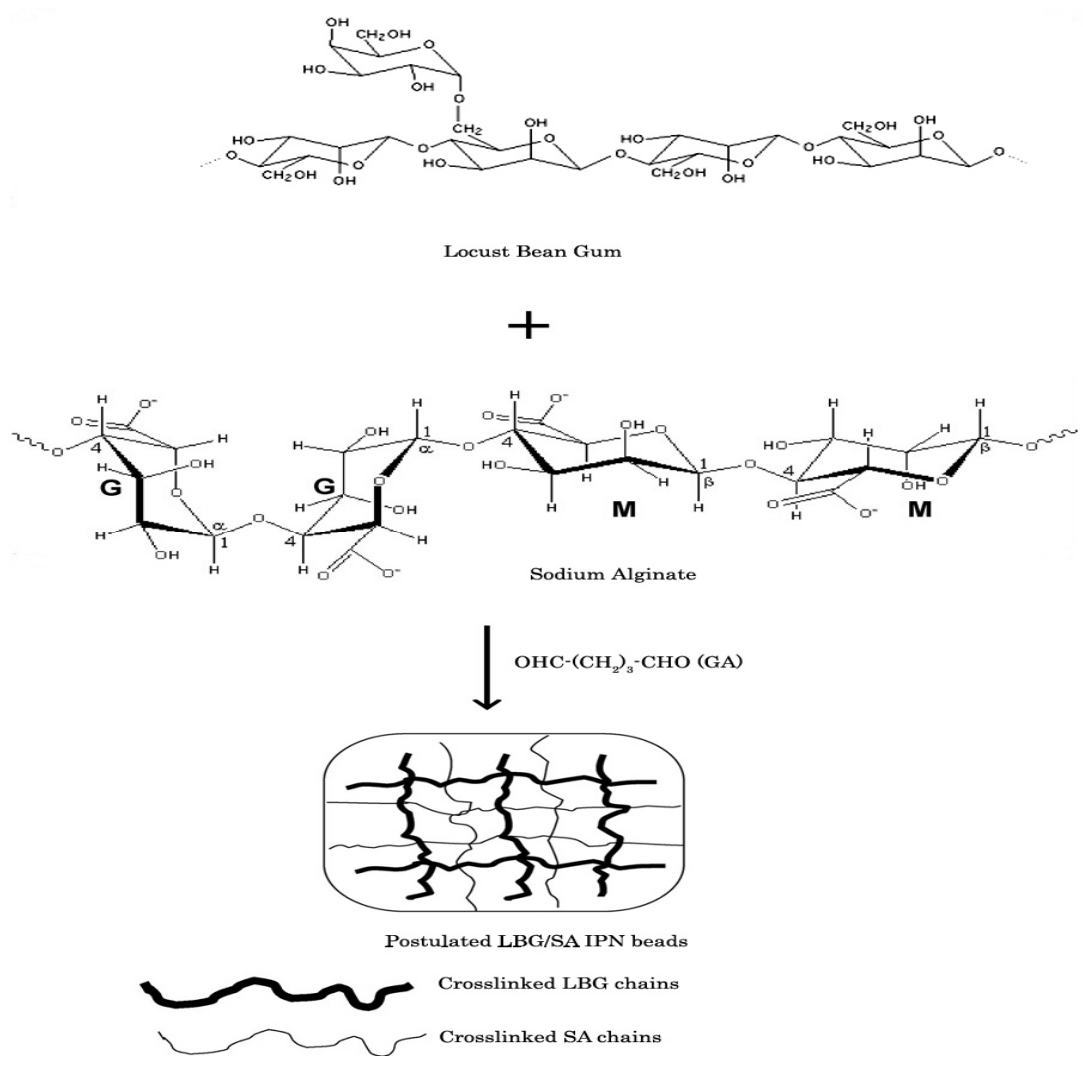

Fig. 1: Formation of IPN structure between LBG and NaAlg 


\section{Differential scanning calorimetry studies}

DSC curves of the plain sodium aliginate-locust bean gum hydrogel beads, plain drug and drug-loaded gel beads were recorded using TA instruments (Model-STA, Q 600 USA). The analysis was performed by heating the sample from 30 to $500{ }^{\circ} \mathrm{C}$ at the rate of $10{ }^{\circ} \mathrm{C} / \mathrm{min}$ under inert atmosphere.

\section{X-Ray diffraction studies}

The X-RD patterns of the plain drug, plain gel beads and drug-loaded gel beads were recorded using a Rigaku Geigerflex Diffractometer (Tokyo, Japan) equipped with Ni-filtered CuK $\alpha$ radiation $(l=1.5418$ $\AA$ ). The dried gel beads of uniform size were mounted on a sample holder and the patterns were recorded in the range $0-50^{\circ}$ at a scanning rate of $5 \%$ min to determine the crystallinity.

\section{Scanning electron microscopic studies}

SEM micrographs of gel beads were obtained under high resolution (magnification, $\times 300 ; 5 \mathrm{kV}$ ) using JOEL MODEL JSM 840A, SEM, equipped with phoenix energy dispersive analysis of EDAX and Leica 400, Cambridge, UK instrument.

\section{Estimation of drug loading and encapsulation efficiency}

The specific amount of drug loaded dry gel beads was vigorously stirred in a beaker containing $10 \mathrm{ml}$ of $7.4 \mathrm{pH}$ phosphate buffer solution to extract the drug from hydrogel beads. The solution was then filtered and analyzed by ultraviolet (UV) spectrophotometer at the $\lambda_{\max }$ of $398 \mathrm{~nm}$. These results of \% drug loading and encapsulation efficiency were calculated using Equations (1) and (2) respectively and are compiled in table 1 .

$$
\begin{aligned}
& \% \text { Drug loading }=\left[\frac{\text { Amount of drug in hydrogelbeads }}{\text { Amount of hydrogel beads }}\right] \times 100 \ldots \ldots . . .(1) \\
& \text { Encapsulation efficiency }=\left[\frac{\text { Actualloading }}{\text { Theoreticalloading }}\right] \times 100 \ldots \ldots \ldots . . .(2)
\end{aligned}
$$

\section{In vitro release studies}

In vitro release studies have been carried out by dissolution experiments using the tablet dissolution tester (Lab India, DS 8000, Mumbai, India) equipped with eight baskets. Dissolution rates were measured at $37{ }^{\circ} \mathrm{C}$ under $100 \mathrm{rpm}$ rotation speed in $600 \mathrm{ml}$ dissolution medium. Drug release from the hydrogel beads was studied in intestinal (7.4 pH phosphate buffer) like fluid. At regular intervals of time, aliquot samples were withdrawn and analyzed using UV spectrophotometer at the fixed max value of $\lambda_{\max }$ at 398 $\mathrm{nm}$. The measurements were taken three times for estimation of standard deviation.

\section{Swelling characteristics of alginate-locust bean gum hydrogel beads}

The swelling characteristics of alginate-LBG hydrogel beads were determined a method described Madhusudana et al., [26]. The dried test samples were immersed in $10 \mathrm{ml}$ distilled water at specific time intervals, the absorbed excess water on the surface of the beads was removed by the gentle touch of tissue paper to measure the swollen sample weight. The swelling ratios (Qs) of test samples were calculated from the equation:

$$
\text { Qs }=\frac{W s-W d}{W d} \times 100 .
$$

Where Ws is the weight of the swollen sample and $\mathrm{Wd}$ is the weight of the dried test sample. The sample which had the best swelling characteristics suitable for the drug delivery among the studied groups was selected for further Nimesulide release profile studies.

\section{RESULTS AND DISCUSSION}

\section{Fourier transform infrared spectroscopy}

FTIR spectra of samples were recorded using FTIR as to ascertain the presence of different functional groups. FTIR spectra analysis of pure Nimesulide (a), Nimesulide-loaded hydrogel beads (b) and placebo hydrogel beads (c) is shown in (fig. 2). The FTIR spectrum of the pure drug (fig. 2a), showed the characteristic peaks at $3425.33 \mathrm{~cm}^{-1}$ due to $\mathrm{N}-\mathrm{H}$ (amide group) stretch, $1581.76 \mathrm{~cm}^{-1}$ for $\mathrm{NO}_{2}$ (aromatic) group, $1354.21 \mathrm{~cm}^{-1}$ for $\mathrm{CH}_{3}$ (alkane group) and $1157.20 \mathrm{~cm}^{-1}$ for $\mathrm{S}=0$ group which revealed the purity of the drug. The FTIR spectrum of drugloaded blend hydrogels (NaAlg/lBG) showed (fig. 2b) characteristic peaks at $3409.90 \mathrm{~cm}^{-1}$ due to stretching of $\mathrm{OH}, 2854.44 \mathrm{~cm}^{-1}$ due to stretching of C-H, $1596.94 \mathrm{~cm}^{-1}$ indicating the bending vibration of $\mathrm{CH}_{2}$ groups, $1126.35 \mathrm{~cm}^{-1}$ showed the $-\mathrm{C}-\mathrm{O}-$ asymmetric stretching frequency, $1072.34 \mathrm{~cm}^{-1}$ related to $\mathrm{C}-\mathrm{O}-\mathrm{H}$ stretching, $763.76 \mathrm{~cm}^{-1}$ and $617 \mathrm{~cm}^{-1}$ due to aromatic groups of plain blend. The FTIR spectrum of placebo blend hydrogel beads (fig. 2c) showed the characteristic peaks at $3764.77 \mathrm{~cm}^{-1}$ due to stretching of- $\mathrm{OH}, 2360.70 \mathrm{~cm}^{-1}$ due to $\mathrm{C}-\mathrm{H}$ stretching, 1126.35 represented C-O-H stretching. These FTIR spectral observations suggest that all functional groups were present in the polymeric hydrogel beads indicating presence of interactions between the drug and blended polymers. A similar observation was reported by Neelam Jain et al.,[30] about the famotidine drug delivery through locust bean gum/PVA polymer network microparticles.

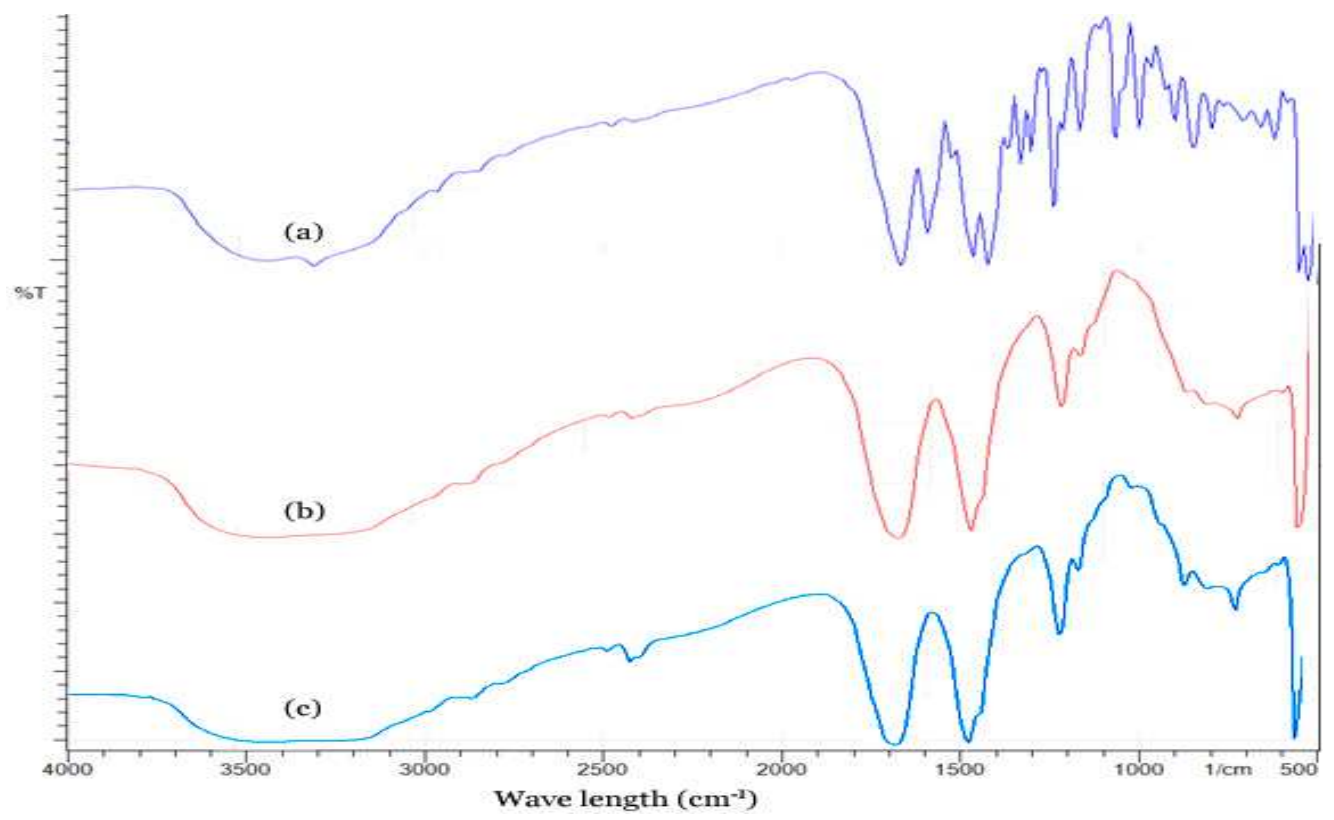

Fig. 2: FTIR spectra analysis of pure Nimesulide (a), Nimesulide loaded blend hydrogels beads (b) and placebo blend hydrogel beads (c) (Results are expressed as mean, $n=3$ ) 


\section{Differential scanning calorimetry}

To study the molecular level dispersion of Nimesulide with polymer IPN hydrogel beads, DSC thermograms were produced for pure Nimesulide (a), Nimesulide loaded blend hydrogel beads (b) and placebo hydrogel beads (c) are shown in (fig. 3).

As shown in (fig. 3a) Nimesulide exhibits a sharp peak at $153.5^{\circ} \mathrm{C}$ corresponding to the melting temperature $\left(150{ }^{\circ} \mathrm{C}\right)$ as reported in the literature Sudhakar et al., [27]. In case of Nimesulide loaded NaAlg-LBG blend hydrogel beads (fig. 3b) the melting temperature peak of Nimesulide was shifted to lower temperature with reduced intensity indicating a significant reduction of the drug crystallinity and was probably marked by the amorphous nature of the blend hydrogel beads.

However, ignoring the appearance of a very small peak at around $153^{\circ} \mathrm{C}$ and the absence of such a peak in placebo (fig. 3c) we can conclude that the drug is molecularly dispersed in the IPN hydrogel beads. This observation is in accordance with the DSC results of Manjanna et al., [31] from the drug release studies of NaAlg-LBG microbeads.

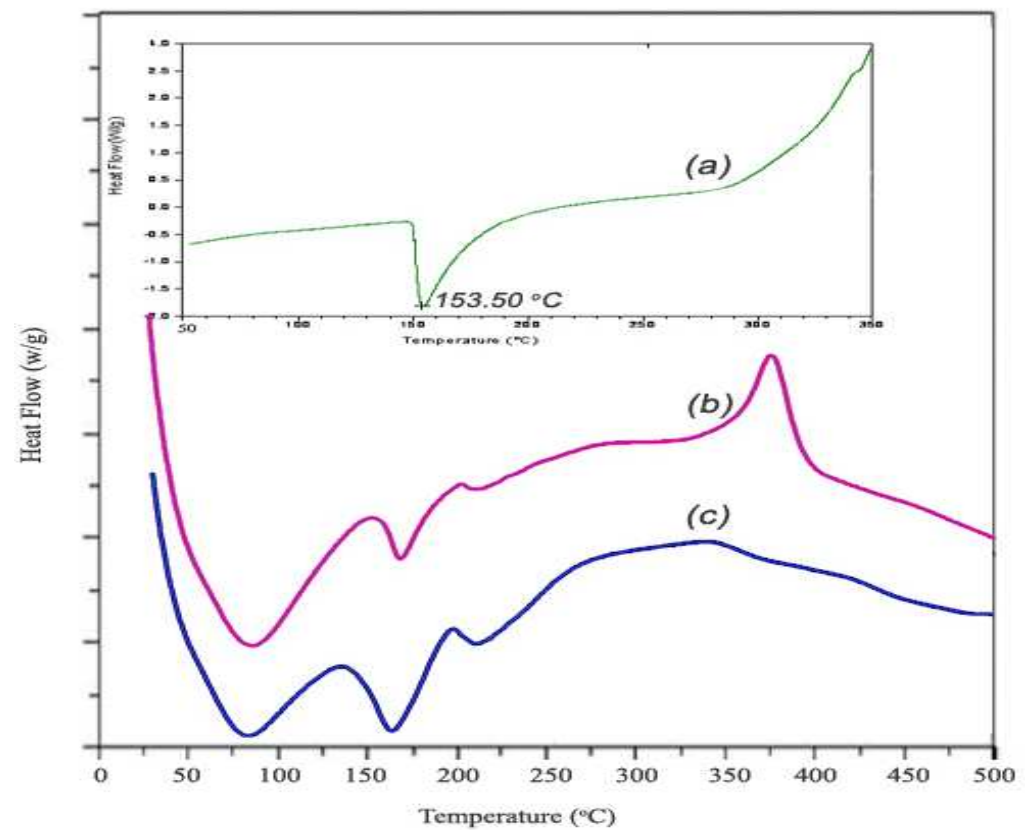

Fig. 3: DSC thermograms of (a) pure drug (Nimesulide) (b) Nimesulide loaded blend hydrogel beads and (c) placebo blend hydrogel beads (Results are expressed as mean, $n=3$ )

\section{Scanning electron microscopy}

Typical SEM images of a placebo drug loaded hydrogel group of beads (fig. 4a) and single hydrogel beads (fig. 4b) are shown in (fig. 4). From (fig. 4a) it is observed that a group of beads and from (fig. 4b) it is noticed the formation of the individual bead. From the SEM images, it is evident that the surface of the hydrogel beads have rough surface in the single bead it was clearly observed (fig. 4b). A similar observation was reported by Aminabhavi et al.,[32] from the isoniazid drug release through blend hydrogel microspheres of NaAlg and PNIPAAm-g-GG.
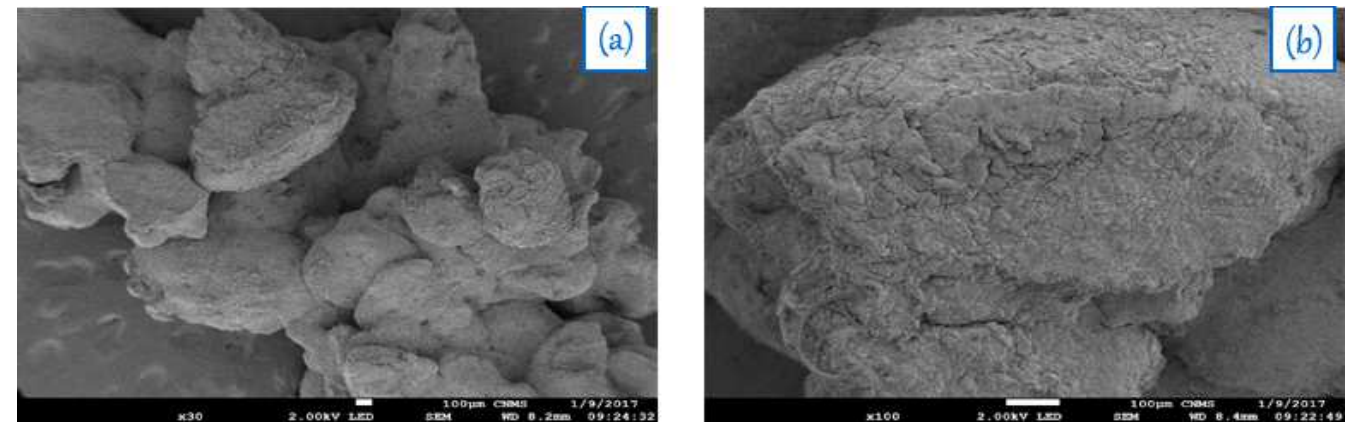

Fig. 4: SEM micrographs of drug loaded IPN hydrogel beads (a) and (b) (Results are expressed as mean, $n=3$ )

\section{$\mathrm{X}$-ray diffractometric analysis}

The presence or absence of crystallinity of the drug and polymer was determined by the X-Ray Diffraction studies by comparing some representative peaks. X-Ray diffractogram of pure Nimesulide drug (a), drug-loaded formulation (b) and placebo formulations (c) are given in (fig. 5). The Nimesulide peaks are observed at $2 \theta$ of $18^{\circ}, 21^{\circ}$ and $29^{\circ}$ suggesting its crystalline nature. In case of placebo blend hydrogel beads, no intense peaks were observed suggesting that it's amorphous nature. But drug loaded hydrogel beads of NaAlg-LBG shows two small peaks appeared at $2 \theta=20^{\circ}$ and $26^{\circ}$ which are possibly due to the surface adhered drug on the polymer blend hydrogel beads. On the other hand, the absence of most of the characteristics peaks of Nimesulide in the drug-loaded hydrogels beads confirms that Nimesulide is molecularly dispersed into the polymer blend matrix. A similar observation was noted by Krishna Rao et al., [33] from the cefedroxil drug release studies through interpenetrating network microgels of chitosan/acrylamide grafted PVA. 


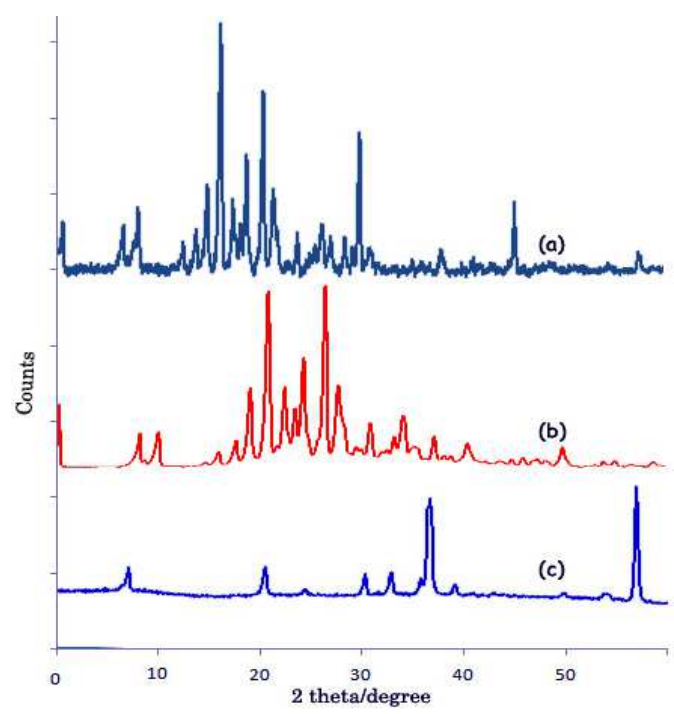

Fig. 5: X-RD patterns of pure Nimesulide (a), Nimesulide loaded IPN hydrogels beads (b) and placebo IP Nhydrogel beads (c) (Results are expressed as mean, $n=3$ )

\section{Swelling behavior}

The swelling index for the various formulations is shown in (fig. 6). These profiles indicate the uptake of water into the hydrogel beads producing an increase in weight. The swelling ratio increased with increasing NaAlg content due to its hydrophilicity. In the case of crosslinker variations, the swelling ratios of hydrogel beads increased, with increasing amount of NaAlg, which enhanced the diffusion of water molecules into the hydrogel beads. The decrease in swelling with decreasing crosslinker was attributed to polymeric chains that become rigid due to the contraction of microvoids. The presence of Nimesulide embedded in the hydrogel caused enlargement of the hydrogel networks. A considerable variation in the swelling capacity was observed when the hydrogel beads were modified or loaded with Nimesulide. The overall results suggested that the dried beads swelled slightly in the acidic medium. When they were subsequently transferred to alkaline medium a thick diffusion layer surrounding the particles was formed, thereby sustaining the release of the incorporated drug.

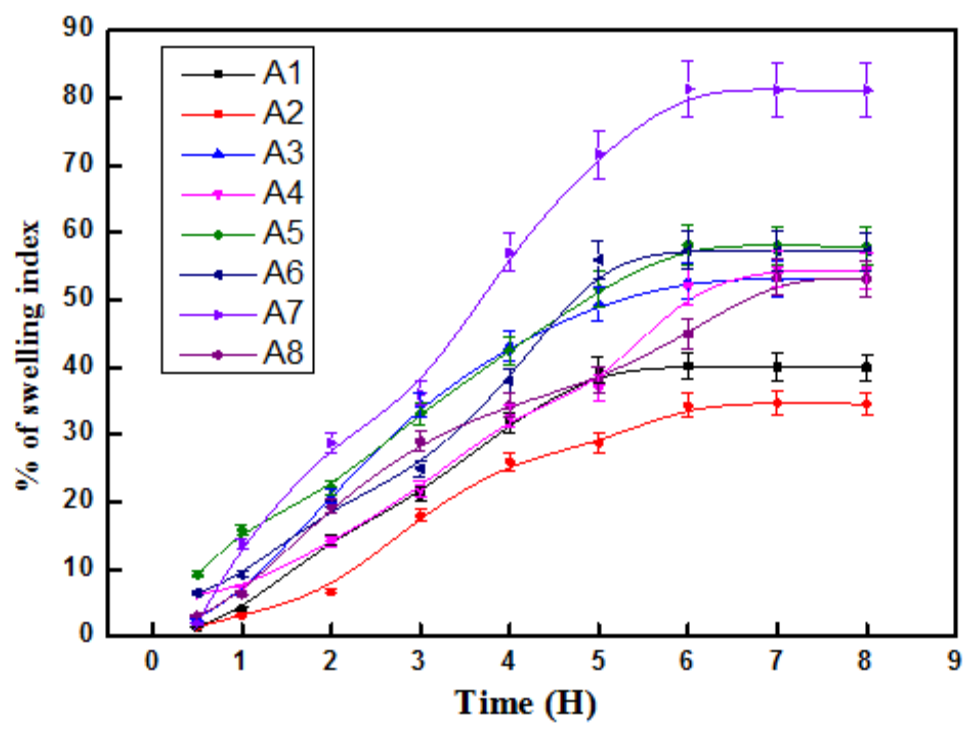

Fig. 6: Comparison of the swelling behavior of hydrogel beads of NaAlg/lBG containing Nimesulide (Results are expressed as mean, n=3)

\section{In vitro release studies}

In vitro drug release data are discussed in terms of the effect of drug loaded, $\mathrm{NaAlg} / \mathrm{lBG}$ blend composition and amount of crosslinking.

\section{Effect of drug loading}

The effect of percentage drug loading on in vitro release for different formulations is displayed in (fig. 7). To study the effect of drug loading on release rates, we have taken the formulations (A4, A5, and A6 with respect to drug loading of $5 \%, 10 \%, 15 \%$ ), wherein A6 shows higher drug release than A5 and A4. This indicates that release rates vary depending upon the amount of drug present in the matrices. During the first $2 \mathrm{~h}$, burst release is observed for all formulations and observed that drug release rates almost same after that the release of drug was extended up to $48 \mathrm{~h}$. Whereas A6 shows around $80 \%$ of drug release, A5 and A4 shows around-76\% of drug release. A similar observation was reported by Patil and Bhoskar [34] from their drug release studies. 


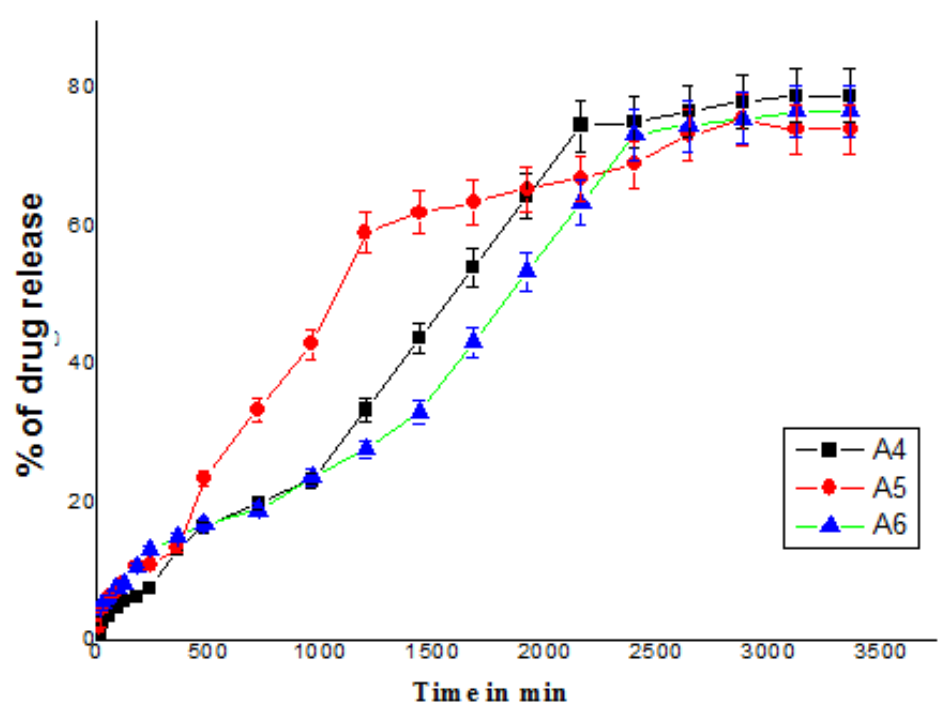

Fig. 7: \% of cumulative release of Nimesulide loaded hydrogel beads containing different amounts of drug (A4-5 \%), (A5-10\%) and (A$15 \%$ ) in $7.4 \mathrm{pH}$ solution (Results are expressed as mean, $\mathrm{n}=3$ )

\section{Effect of crosslinking}

Fig. 8 displays the in vitro release profiles of hydrogel beads crosslinked with different amounts of GA containing $1.0 \mathrm{ml}, 1.5 \mathrm{ml}, 2.0 \mathrm{ml}$ (i.e., formulations A7, A4 and A8). Cumulative (\%) drug release is higher in the case of hydrogel beads crosslinked with $1 \mathrm{ml}$ of GA. It may be noted that the release rates increased with decreasing cross linking because the loose matrices allow faster diffusion of the drug into the dissolution media. This may be due to the fact that at higher crosslinks, the free volume of the matrix will be reduced, thereby hindering easy transport of drug molecules through the matrix. The drug was much similar in all formulations. However, in all formulations, the burst effect is observed, but the release was extended up to $48 \mathrm{~h}$. Similar results were reported in literature [35].

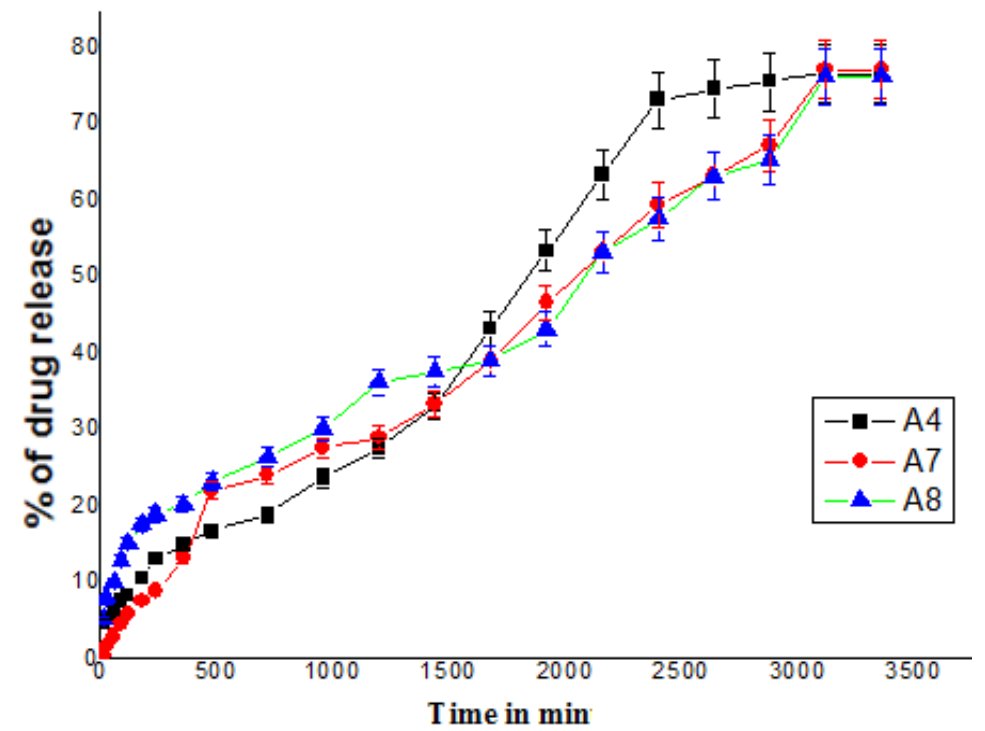

Fig. 8: \% of cumulative release of Nimesulide through hydrogel beads containing different amounts of crosslinker formulations A7 (1 ml), A4 (1.5 ml) and A8 (2 ml) in pH7.4 solution (Results are expressed as mean, $\mathrm{n}=3$ )

\section{Effect of polymer variation}

The cumulative percentage drug release from Nimesulide loaded hydrogel beads were found sustained over a period of $50 \mathrm{~h}$ (fig. 9). The percentage drug released from Nimesulide loaded NaAlgLBG hydrogel beads in $24 \mathrm{~h}$ was within the range of $30-40 \%$. As the polymer (LBG) concentration increased, the release rate of Nimesulide from the hydrogel beads was decreased. The slower release rate can be explained by the increase in the extent for swelling and rate can be explained by the increase in the extent for swelling and the gel layer thickness that acted as a barrier for the penetration medium thereby retarding the diffusion of the drug from the swollen beads. The results also explained that the initial burst release of drug was reduced by increasing the concentration of LBG because of formation of diffusional bridges due to swelling of hydrophilic linkage. A similar observation was made by Durgapal et al. [36] from their Ciprofloxacin drug delivery studies. 


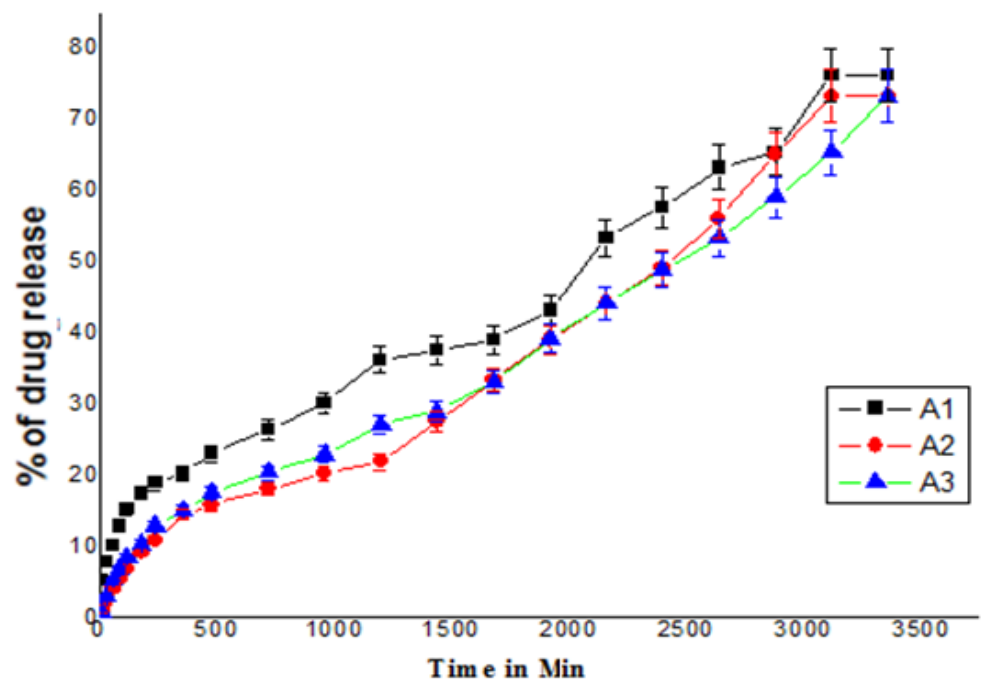

Fig. 9: \% Cumulative release of Nimesulide loaded hydrogel beads for different ratios of polymer composition (A1, A2, and A3) (Results are expressed as mean, $n=3$ )

\section{Drug release kinetics}

Drug release kinetics was analyzed by plotting the cumulative release data Vs time by fitting to the empirical equation [37].

$$
\mathrm{M}_{\mathrm{t}} / \mathrm{M}_{\alpha}=\mathrm{kt}^{\mathrm{n}} \ldots \ldots \ldots \ldots \ldots \text { (4) }
$$

Here $\mathrm{M}_{\mathrm{t}} / \mathrm{M}_{\alpha}$ represents the fractional drug release at time $\mathrm{t}, \mathrm{k}$ is a constant characteristic of the drug-polymer system, and $n$ is an empirical parameter characterizing the release mechanism. Using the least squares procedure, we have estimated the values of $\mathrm{n}$ and $\mathrm{k}$ for all the eight formulations and these values are given in table 2. If $\mathrm{n}=0.5$, then drug diffuses and releases from the polymer matrix following a Fickian diffusion. For $n>0.5$, anomalous or non-Fickian is operative. The intermediary values ranging between 0.5 and 1.0 are attributed to the anomalous type transport [23].
The values of $\mathrm{k}$ and $\mathrm{n}$ have shown a dependence on the extent of crosslinking, \% drug loading and polymer blend composition. Values of $n$ for hydrogel beads prepared by varying the amount of LBG in the beads of $0.25,0.5$ and 0.75 by keeping Nimesulide (5\%) and GA(1.5 ml) constant ranged from 0.493 to 0.678 , leading to a shift of transport from Fickian to anomalous type. The Nimesulide loaded hydrogel beads varying the amount of drug (Nimesulide) (5\%, 10\%, $15 \%)$ by keeping blend ratio (3:1) and GA (1.5) concentration have the ' $n$ ' values ranging from 0.621 to 0.769 , indicating the shift from erosion type release to a swelling-controlled non-Fickian mechanism. This is because of a reduction in the regions of low microviscosity and closure of micro activities in the swollen state of the IPN matrix. Similar findings have been observed by Jana et al., [38] wherein the effect of different polymer ratios on dissolution kinetics was studied.

Table 2: Results of \% of release kinetics parameters $(k$, and $n)$ of drug in different IPN hydrogel beads formulations

\begin{tabular}{lll}
\hline Formulation code & $\mathbf{p H ~ 7 . 4}$ & $\mathbf{k}$ \\
\cline { 2 - 3 } & $\mathbf{n}$ & 4.98 \\
$\mathrm{~A} 1$ & 0.493 & 4.98 \\
A2 & 0.562 & 4.58 \\
A3 & 0.678 & 3.15 \\
A4 & 0.621 & 3.52 \\
A5 & 0.648 & 3.88 \\
A6 & 0.769 & 3.56 \\
A7 & 0.591 & 4.37 \\
A8 & 0.654 & \\
\hline
\end{tabular}

\section{CONCLUSION}

Nimesulide-loaded NaAlg/IBG IPN hydrogel beads were prepared through ionotropic gelation technique and studied for drug release. The drug encapsulation efficiency of these hydrogel beads was found to be $42.58 \%$ to $74.23 \%$. FTIR was used to confirm the formation of an interpenetrating network between polymer and drug. DSC analysis of the drug-loaded hydrogel beads confirmed the molecular level dispersion of the drug in the blend hydrogel beads. The in vitro dissolution showed the controlled release of Nimesulide up to $48 \mathrm{~h}$ and followed the Korsmeyer-Peppas model for diffusion studies. These results clearly demonstrated that the ability of these newly developed hydrogel beads containing Nimesulide for its sustained release could possibly be advantageous in terms of advantageous to patient compliance with reduced dosing interval.

\section{ACKNOWLEDGEMENT}

The authors thank UGC, New Delhi for the financial support given in the form of fellowship and is gratefully acknowledged.

\section{AUTHORS CONTRIBUTION}

1. Chintha Madhavi, Areti Parandhama and 0. Sreekanth Reddy as authors are involved in designing of the work and data collection and data analysis.

2. Y. Maruthi and P. Kumar babu as co-authors are involved in the planning of the experiment and data interpretation and drafting $f$ the article.

3. M. C. S. Subha, K. Chowdoji Rao and R. Jeevan Kumar are involved in final drafting and critical revision of the article in the final form. 


\section{CONFLICT OF INTERESTS}

The authors declare that they have no conflict of interest

\section{REFERENCES}

1. Tabata Y, Ikada Y. Synthesis of gelatin microspheres containing interferon. Pharm Res 1989;6:422-7.

2. Peppas N, Burns P, Leobandung W, Chikawa HI. Hydrogels in pharmaceutical formulations. Eur J Pharm Biopharm 2000;50:27-46.

3. Sperling LH. Interpenetrating polymer networks and related materials. Plenum Press: New York; 1981.

4. Dave AM, Mehta MH, Aminabhavi TM, Kulkarni AR, Soppimath KS. Review on controlled release of nitrogen fertilizers through polymeric membrane devices. Polym Plast Technol 1999;38:675-711.

5. Dong LC, Hoffman AS. A novel approach for the preparation of pH-sensitive hydrogels for enteric drug delivery. J Controlled Release 1991;15:141-52.

6. Peppas NA, Korsmeyer RW. Hydrogels in medicine and pharmacology, Boca Raton, FL: CRC Press; 1987.

7. Seigel RA, Firestone BA. Mechanochemical to self-regulating insulin pump design. J Controlled Release 1990;11:181-92.

8. Davis TP, Huglin MB. Effect of composition on properties of copolymeric N-Vinyl-2-pyrrolidone/methyl methacrylate hydrogels and organogels. Polymer 1990;31:513-9.

9. Desai NP, Hubbell JA. Surface physical interpenetrating networks of poly(ethylene terephthalate) and poly(ethylene oxide) with biomedical applications. Macromolecules 1992;25:226-32.

10. Khare AR, Peppas NA. Investigation of hydrogel water in polyelectrolyte gels using differential scanning calorimetry. Polymer 1993;34:4595-800.

11. Burugapalli K, Bhatia D, Koul V, Choudhary V. Interpenetrating polymer networks based on poly(acrylic acid) and gelatin, I: Swelling and thermal behavior. J Appl Polym Sci 2001;82:217-27.

12. Changez M, Koul V, Burugapalli K, Dinda AK. Studies on biodegradation and release of gentamicin sulphate from interpenetrating polymer networks hydrogels based on poly(acrylic acid) and gelatin: In vitro and in vivo. Biomaterials 2004;25:139-46.

13. Hijorth HT, Jan K. Alginate in drug delivery systems. Drug Dev Ind Pharm 2002;28:621-30.

14. Dey P, Maiti S, Sa B. Locust bean gum and its application in pharmacy and biotechnology: an overview. Int J Curr Pharm Res 2012;4:7-11.

15. Dea ICM, Morrison A. Chemistry and Interactions of seed galactomannans. Adv Carbohydr Chem Biochem 1975;31:242312.

16. Davis R, Brogden RN. Nimesulide: an update of its pharmacodynamic and pharmacokinetic properties and therapeutic efficacy. Drugs 1994;48:431-54.

17. Meriani F, Coceani N, Sirotti C, Voinovich V, Grassi M. Characterization of a quaternary liquid system improving the bioavailability of poorly water-soluble drugs. J Colloid Interface Sci 2003;263:590-6.

18. Piel G, Pirotte B, Delneuveille I, Neven P, Delarge J. Study of the influence of both cyclodextrins and l-iysine on the aqueous solubility of Nimesulide: isolation and characterization of Nimesulide-L-Iysine-cyclodextrin complexes. J Pharm Sci 1997;86:475-80.

19. Ferreira SH. Role of interleukins and nitric oxide in the mediation of inflammatory pain and its control by peripheral analgesics. Drugs 1993;46:1-9.

20. Tognell S. New clinical opportunities. Drugs 1993;46:275-6.

21. Ravikumara NR, Madhusudhan B, Nagaraj TS, Hiremat SR, Raina G. Preparation and evaluation of Nimesulide-loaded ethylcellulose and methylcellulose nanoparticles and microparticles for oral delivery. J Biomater Appl 2009;24:47-64.

22. Dutet J, Lahiani MS, Didier L, Jezequel S, Bounoure F, Barbot C. Nimesulide/cyclodextrin/PEG 6000 temary complexes: Physico-chemical characterization, dissolution studies and bioavailability in rats. J Inclusion Phenom Macrocyclic Chem 2007;57:203-9.

23. Singla AK, Chawla $M$, Singh A. Nimesulide: some pharmaceutical and pharmacological aspects and update. J Pharm Pharmacol 2000;52:467-86.

24. Paul AL, Hardman JG, Limbird LE, Molinoff PB, Ruddon WR, Gilman AG. editors. Goodman and Gilman's The Pharmacological basis of therapeutics, chapter, Drugs Affecting Renal and Cardiovascular Function. $9^{\text {th }}$ ed. New York: McGrawHill Companies. Inc: 1996. p. 644.

25. Lakshminarayana Reddy C, Yerriswamy B, Prasad CV, Subha MCS, Chowdojirao K. Control release of chlorpheniramine maleate through IPN beads of sodium alginate-g-methyl methacrylate. J Appl Polym Sci 2010;118:2342-9.

26. Madhusudana Rao K, Krishna Rao KSV, Ramanjaneyulu G, Chowdoji Rao K, Subha MCS, Chang-Sik Ha. Biodegradable sodium alginate-based semi-interpenetrating polymer network hydrogels for antibacterial application. J Biomed Mater Res 2014;102:3196-206.

27. Sudhakar K, Madhusudana Rao K, Mallikarjuna B, Prasad CV, Subha MCS, Chowdoji Rao K. Preparation and characterization of Nimesulide loaded poly(methyl methacrylate)

28. /poly(ethylene oxide) blend microspheres: in vitro release studies. Asian J Pharm 2013;7:118-24.

29. Kumarababu P, Maruthi Y, Veerapratap S, Sudhakar K, Rotimi Sadiku, Prabhakar MN, Jung Il Song, et al. Development and characterization of polycaprolactone (PCL)/poly ((r)-3hydroxybutyric acid) (PHB) blend microspheres for tamoxifen drug release studies. Int J Pharm Pharm Sci 2015;7:95-100.

30. Siraj S, Sudhakar P, Sajankumarji Rao U, Sekharnath KV, Chowdoji Rao K, Subha MCS. Interpenetrating polymer network microspheres of poly (vinyl alcohol)/methyl cellulose for controlled release studies of 6-thioguinine. Int J Pharm Pharm Sci 2014;6:101-6.

31. Jain N, Kumar H, Rajpoot AK, Verma HC. Novel interpenetrating polymer network micro adhesive microspheres of locust bean gum and poly(vinyl alcohol) for the delivery of famotidine. MIT Int J Pharm Sci 2015;1:27-36.

32. Manjanna KM, Rajesh KS, Shiva Kumar B. Formulation and optimization of Natural polysaccharide hydrogel microbeads of aceclofenac sodium for oral controlled drug delivery. Am J Med Sci 2013;1:5-17.

33. Kajjari PB, Manjeswar LS, Aminabhavi TM. Nevel pH and temperature responsive blend hydrogel Microsphere of sodium alginate and PNIPAAm-g-GG for controlled release of isoniazid. AAPS PharmaSciTech 2012;13:1147-57.

34. Krishna Rao KS V, Vijaya Kumar Naidu B, Subha MCS, Sairam M, Aminabhavi T M. Novel chitosan-based $\mathrm{pH}$ sensitive interpenetrating network microgels for controlled release of cefadroxil. Carbohydr Polym 2006;66:333-44.

35. Patil P, Bhoskar M. Optimization and evaluation of spray dried chitosan nanoparticles containing doxorubicin. Int J Curr Pharm Res 2014;6:7-15.

36. Bajpai AK, Rajpoot $M$. Release and diffusion of sulfamethoxazole through acrylamide-based hydrogel. J Appl Poly Sci 2001;81:1238-47.

37. Durgapal S, Mukhopadhyay S, Goswami L. Preparation, characterization and evaluation of floating microparticles of ciprofloxacin. Int J Appl Pharm 2017;9:1-8.

38. Jacobsen J. Buccal iontophoretic delivery of atenolol: $\mathrm{HCl}$ employing a new in vitro three-chamber permeation cell. J Controlled Release 2001;70:83-95.

39. Jana S, Gandhi A, Sheet S, Kumar Sen K. Metal ion-induced alginate-locust bean gum IPN microspheres for sustained oral delivery of aceclofenac. Int J Biol Macromol 2015;72:45-53.

\section{How to cite this article}

C Madhavi, P Kumara Babu, Y Maruthi, A Parandhama, O Sreekanth Reddy, K Chowdoji Rao, MCS Subha, R Jeevan Kumar. Sodium alginate-locust bean gum IPN hydrogel beads for the controlled delivery of the Nimesulide-anti-inflammatory drug. Int J Pharm Pharm Sci 2017;9(10):245-252. 